\title{
Impacto en el aroma de vinos Tannat producidos por diferentes levaduras en tres sistemas de vinificación
}

\section{Impact on the aroma of Tannat wines produced by different yeasts in three winemaking systems}

DOI: $10.46932 / \mathrm{sfjdv} 2 \mathrm{n} 2-034$

Received in: january 1st, 2020

Accepted in: March 30th, 2020

\section{Karina Medina \\ Biology Doctor}

Universidad de la República, Facultad de Química, Área Enología y Biotecnología de Fermentaciones,

Montevideo, Uruguay, General Flores 2124

E-mail: kmedina@fq.edu.uy

\section{Eduardo Boido}

Chemistry Doctor

Universidad de la República, Facultad de Química, Área Enología y Biotecnología de Fermentaciones, Montevideo, Uruguay, General Flores 2124

E-mail: ebloido@fq.edu.uy

\section{Laura Fariña}

Chemistry Doctor

Universidad de la República, Facultad de Química, Área Enología y Biotecnología de Fermentaciones, Montevideo, Uruguay, General Flores 2124

E-mail: lfarina@fq.edu.uy

\section{Gastón Ares}

Chemistry Doctor

Universidad de la República, Facultad de Química, Instituto Polo Tecnológico de Pando, Sensometría y Ciencias del Consumidor, Canelones, Uruguay

By Pass de Pando y Ruta 8

E-mail: gares@fq.edu.uy

\section{Eduardo Dellacassa}

Chemistry Doctor

Universidad de la República, Facultad de Química, Área Enología y Biotecnología de Fermentaciones, Montevideo, Uruguay, General Flores 2124

E-mail: edellac@fq.edu.uy

\section{Francisco Carrau \\ Biology Doctor}

Universidad de la República, Facultad de Química, Área Enología y Biotecnología de Fermentaciones,

Montevideo, Uruguay, General Flores 2124

E-mail: fcarrau@fq.edu.uy 


\title{
RESUMEN
}

Las vinificaciones realizadas consistieron en cultivos puros de Saccharomyces cerevisiae, y cultivos mixtos con inoculación secuencial de una cepa Hanseniaspora vineae (T02/5AF) y Hanseniaspora clermontiae (A10/82F), con una cepa convencional de Saccharomyces cerevisiae. Los sistemas de vinificación realizados con uvas Tannat fueron: semipiloto, piloto y escala industrial. Fueron identificados 51 compuestos volátiles De un total de 51 compuestos volátiles cuantificados para el total de las vinificaciones, se encontraron 16 compuestos con unidades de aromas mayores a la unidad, y por lo tanto con potencial contribución en el perfil sensorial. La caracterización sensorial aromática se realizó mediante la técnica de mapeo proyectivo, con 48 consumidores que realizaron la evaluación sensorial del total de los tratamientos para las tres vinificaciones. El análisis factorial múltiple realizado permitió comparar los resultados químicos y sensoriales con el fin de encontrar correlaciones. Se encontró que existió concordancia entre ambos estudios lo que confirma las diferentes tendencias aromáticas encontradas en las diferentes vinificaciones. En ambos estudios se observó que las vinificaciones semipiloto estuvieron caracterizadas por los descriptores "químico" y "floral", las vinificaciones piloto por el descriptor "especiado", y las vinificaciones industriales por "frutado" y "madera".

Palabras clave: Levadura, Aroma, Fermentación, Tannat, Vino

\begin{abstract}
The vinifications carried out consisted of pure cultures of Saccharomyces cerevisiae, and mixed cultures with sequential inoculation of a strain Hanseniaspora vineae (T02/5AF) and Hanseniaspora clermontiae (A10/82F), with a conventional strain of Saccharomyces cerevisiae. The vinification systems carried out with Tannat grapes were: semi-pilot, pilot and industrial scale. Of a total of 51 volatile compounds quantified for the total number of vinifications, 16 compounds were found with aroma units greater than unity, and therefore with a potential contribution to the sensory profile. Aromatic sensory characterization was performed using the projective mapping technique, with 48 consumers performing the sensory evaluation of the total treatments for the three vinifications. The multiple factorial analysis carried out allowed the chemical and sensory results to be compared in order to find correlations. It was found that there was concordance between the two studies, which confirms the different aromatic tendencies found in the different vinifications. In both studies it was observed that the semi-pilot vinifications were characterized by the descriptors "chemical" and "floral", the pilot vinifications by the descriptor "spicy", and the industrial vinifications by "fruity" and "woody".
\end{abstract}

Key words: Yeast, Aroma, Fermentation, Tannat, Wine.

\section{INTRODUCCIÓN}

El umbral de percepción de los compuestos volátiles presentes en el vino es muy variado, y esto determina que el impacto de los distintos compuestos volátiles en el aroma dependa de las unidades de aroma, que se definen como la relación entre la concentración de una sustancia y el umbral de percepción de la misma. Por esta razón, compuestos presentes en un vino en concentraciones del orden de ng/L pueden tener un impacto notable en el aroma, mientras que otros con concentraciones de varios cientos de $\mathrm{mg} / \mathrm{L}$ pueden prácticamente no influir en las características sensoriales de la muestra, dependiendo de sus respectivos umbrales de percepción [1]. En muchos casos existe dificultad de obtener los valores de umbral de percepción para un importante número de compuestos volátiles, y por otro lado, el tema se 
complejiza aún más, ya que el impacto de los compuestos en forma individual no es el percibido sensorialmente debido a las interacciones que aparecen en mezclas complejas como son las bebidas y en especial el vino [2]. Por otro lado, hay que considerar que los diferentes sistemas de vinificación impactan en los aromas y la percepción de los mismos, debido a factores tales como temperatura, humedad relativa, cantidad de oxígeno disponible, velocidad de sedimentación de las células, etc, [3]. Esto, sumado a que el estudio de las levaduras no-Saccharomyces, ya sea en cultivo puro como mixto, ha sido estudiado fundamentalmente a escala de laboratorio y dado que los resultados efectivamente no son los mismos de lo que se puede esperar de vinificaciones industriales [4], surge la necesidad de abordar el tema contemplando diferentes sistemas de vinificación.

En este trabajo, se estudió la posible contribución de los distintos compuestos volátiles, en el perfil sensorial de los vinos obtenidos en tres sistemas diferentes de vinificación, a escala semipiloto, piloto e industrial, y su impacto en las unidades de aroma.

\section{MATERIALES Y MÉTODOS}

\subsection{CEPAS DE LEVADURA.}

Las levaduras utilizadas para las diferentes vinificaciones fueron las levaduras nativas Hanseniaspora vineae Hv T02/05F, Hanseniaspora clermontiae Hc A10/82F, Saccharomyces cerevisiae Sc 882 y levaduras comerciales de laboratorio Lallemand ALG 804, ALG 811 y Torulaspora delbrueckii. Todos los ensayos se realizaron con uvas Tannat, con un tamaño de inóculo de $1 \times 10^{6} \mathrm{cel} / \mathrm{mL}$ [5]. Los cultivos mixtos se realizaron con inoculación secuencial a las 72 horas.

\subsection{VINIFICACIONES REALIZADAS.}

Las modalidades de vinificación fueron las siguientes:

I) Escala semipiloto en tarrinas de 20 litros, con tres tratamientos: cultivo mixto $\mathrm{Hv} \mathrm{T} 02 / 05 \mathrm{~F}+\mathrm{Sc} 882$ $(\mathrm{Hv}+\mathrm{Sc} \mathrm{M})$; cultivo mixto Hc A10/82F + Sc $882(\mathrm{Hc}+\mathrm{Sc} \mathrm{M})$ y cultivo puro Sc $882(\mathrm{Sc} \mathrm{M})$

II) Escala piloto en bins de 500 litros, con 2 tratamientos: cultivo mixto Hv T02/05F + Sc ALG 804 $(\mathrm{Hv}+\mathrm{Sc} \mathrm{C})$ y cultivo puro con Sc ALG 804 (Sc C)

III) Escala industrial en tinas de roble de 5.000 litros, con 3 tratamientos: cultivo mixto Hv T02/05F + Sc ALG $111(\mathrm{Hv}+\mathrm{Sc} \mathrm{B})$, cultivo mixto Torulaspora delbrueckii (comercial) + Sc ALG $111($ Torul + Sc B) y cultivo puro Sc ALG 111 (Sc B) 


\subsection{ANÁLISIS DE AROMAS.}

Para cada una de las vinificaciones se realizó la extracción de compuestos aromáticos mediante SPE (ISOLUTE® ENV+) según Carlin (1998) [6], y puesto a punto para vinos Tannat por Boido et al. [7]. La identificación se realizó mediante GC-MS Shimadzu GC-17 acoplado con un detector de espectrofotometría de masa Shimadzu QP 5050, y la cuantificación por medio de HRGC, en un cromatógrafo GC-14B, equipado con detector de ionización de llama (FID) y software de procesamiento de datos Shimadzu EZ Chrom, versión 3.2 [8].

\subsection{CARACTERIZACIÓN AROMÁTICA SENSORIAL.}

Para todas las vinificaciones se realizó una caracterización sensorial por Mapeo Proyectivo o Napping ${ }^{\circledR}$ [9], que permitió cuantificar las percepciones de similitud y diferencia globales entre las muestras, obteniéndose una aproximación al perfil sensorial y sin necesidad de contar con una descripción muy detallada de las características sensoriales [10]. El mapeo proyectivo se ha aplicado a una amplia gama de alimentos y bebidas, incluyendo vinos [11-12]. En cada uno de los estudios se presentó un set conteniendo las 8 muestras de forma simultánea en orden aleatorio. Se solicitó a los 48 consumidores participantes del estudio, que olieran las muestras y que las ubicaran en una hoja de papel de $40 \mathrm{x} 30 \mathrm{~cm}$, de acuerdo al grado de diferencia o similitud entre ellas y se les solicitó que indicaran las palabras que consideraban adecuadas para describir cada una de las muestras. Para cada mapa proyectivo, se determinaron las coordenadas $\mathrm{X}$ e $\mathrm{Y}$ de cada muestra, considerando como origen del sistema de coordenadas el extremo inferior izquierdo de la hoja. Las evaluaciones se realizaron en una sala de evaluación normalizada según norma ISO 8589 [13].

\subsection{ANÁLISIS ESTADÍSTICO.}

Se realizó análisis de varianza de los datos de concentración de aromas, considerando cepa como factor de variación y nivel de significación 5\%. Cuando las diferencias fueron significativas se aplicó el test de Tukey para determinación de diferencias entre tratamientos. Se realizó además un análisis de componentes principales (PCA). Estos análisis se realizaron utilizando el software Statistica 7.0 (Stat Soft, Inc., Tulsa, USA). Los datos de análisis sensorial obtenidos por Napping, fueron analizados utilizando Análisis Factorial Múltiple (MFA), según Pagès (2005) [11], y realizados en el software R (R Core Team, 2013) utilizando el paquete FactoMineR [14]. 


\section{RESULTADOS Y DISCUSIÓN}

En la tabla 1, se presentan los compuestos aromáticos encontrados en los vinos, para los que la unidad de aroma fue igual o mayor a 1 en algunos de los tratamientos, y su correspondiente descriptor y umbral de percepción. De un total de 51 compuestos volátiles cuantificados para el total de las vinificaciones, se encontraron 16 compuestos con unidades de aromas mayores a la unidad, y por lo tanto con potencial contribución en el perfil sensorial. Dentro de estos compuestos, no se encontró ninguno del grupo de los ácidos, isoácidos ni norisoprenoides. Dentro del grupo de los alcoholes, se destacaron el alcohol isoamílico y el alcohol $\beta$-feniletílico. Para ambos compuestos, los tratamientos con S. cerevisiae presentaron valores mayores de UA que los respectivos tratamientos con $H$. vineae y $H$. clermontiae. La contribución sensorial del 1-hexanol solo estuvo presente en los tratamientos de la vinificación semipiloto. Para los ésteres etílicos, el hexanoato de etilo es el compuesto que se destacó por los valores elevados de UA en todos los tratamientos. En la vinificación semipiloto, se destacó el valor elevado de las UA de la cepa Sc 882. Esta tendencia de mayores UA por parte de $S$. cerevisiae sobre $H$. vineae se observó para la vinificación semipiloto e industrial. Para esta última, el mayor valor de UA correspondió al tratamiento con la cepa Torulaspora. Entre los acetatos, el acetato de isoamilo y el acetato de $\beta$-feniletilo, fueron los compuestos con mayores UA en todos los tratamientos. Para el acetato de etilo, se observó por parte de los cultivos mixtos con las levaduras no-Saccharomyces, un mayor aporte sensorial, destacándose el tratamiento con las levaduras H. clermontiae + Sc. Para las lactonas, el único aporte sensorial estuvo dado por la $\gamma$-butirolactona, la que a su vez solo estuvo presente en el tratamiento puro con S. cerevisiae de la vinificación piloto. Dentro de los fenoles volátiles, el guaicol fue el que presentó los valores mayores de UA para todos los tratamientos.

Si a partir de los datos obtenidos en la tabla 1, se realiza una agrupación general de los compuestos en función de los descriptores (sin tomar en cuenta a que grupo químico pertenecen), se observa que el descriptor primario "frutal" y "químico" reunieron el mayor grupo de compuestos respectivamente. Bajo el descriptor primario "frutal", se encontró el acetato de isobutilo, acetato de isoamilo, acetato de $\beta$ feniletilo, butirato de etilo y hexanoato de etilo. Para el descriptor primario "químico", se encontró el acetaldehído, alcohol isoamílico, alcohol isobutílico, acetato de etilo y metionol. Contrariamente, los descriptores primarios "vegetal", "floral" y "especiado", estuvieron representados de manera general en el total de las vinificaciones realizadas por un solo compuesto respectivamente, 1-hexanol para "vegetal", alcohol $\beta$-feniletílico para "floral", y 4-vinil-guaicol para "especiado". En cuanto a la caracterización aromática mediante la técnica de mapeo proyectivo, se encontró que existió concordancia entre ambos estudios lo que confirma las diferentes tendencias aromáticas encontradas en las diferentes vinificaciones. En ambos estudios se observó que las vinificaciones semipiloto estuvieron caracterizadas 
por los descriptores "químico" y "floral"; las vinificaciones piloto por el descriptor "especiado"; y las vinificaciones industriales por "frutado" y "madera". Esta comparación demostró la existencia de correlación entre el estudio químico de las UA realizado a partir de los umbrales de percepción de los diferentes compuestos y la evaluación sensorial realizada con consumidores.

\section{CONCLUSIONES}

La aplicación de las diferentes cepas produjo cambios en la composición química de los compuestos volátiles de los vinos obtenidos. Esto se demostró a nivel cromatográfico por las diferencias encontradas en la composición de la fracción volátil y posteriormente por las diferencias encontradas en el perfil sensorial que reflejo la contribución sensorial de las diferentes cepas al aroma de los vinos Tannat elaborados. A su vez, el efecto del tipo de manejo en la vinificación, también produjo importantes cambios en la composición aromática. Se pudo demostrar que existió correlación entre el estudio químico de suma de unidades de aromas con igual descriptor y el estudio sensorial realizado con consumidores.

Tabla 1. Cálculo de las unidades de aroma (UA), para los distintos compuestos volátiles presentes en las tres vinificaciones realizadas. (*) Medio en el que se determina el umbral de percepción: a, agua; sv, simil vino; [2]. (ref): [15].

\begin{tabular}{|c|c|c|c|c|c|c|c|c|c|c|}
\hline Compuesto & Descriptor & Umbral $(\mu \mathrm{g} / \mathrm{L})^{\star}$ & $\begin{array}{c}\mathrm{Hc}+\mathrm{Sc} \mathrm{M} \\
\text { UA }\end{array}$ & $\begin{array}{c}\mathrm{Hv}+\mathrm{Sc} \mathrm{M} \\
\text { UA }\end{array}$ & $\begin{array}{c}\text { Sc M } \\
\text { UA }\end{array}$ & $\begin{array}{c}\mathrm{Hv}+\mathrm{ScC} \\
\text { UA }\end{array}$ & $\begin{array}{l}\text { Sc C } \\
\text { UA }\end{array}$ & $\begin{array}{c}\mathrm{Hv}+\mathrm{Sc} \mathrm{B} \\
\text { UA }\end{array}$ & $\begin{array}{l}\text { Sc B } \\
\text { UA }\end{array}$ & $\begin{array}{c}\text { Torul +Sc B } \\
\text { UA }\end{array}$ \\
\hline 1-hexanol & $\begin{array}{l}\text { pasto recien } \\
\text { cortado }\end{array}$ & $2500 \mathrm{sv}$ & 1 & 1 & 1 & $<1$ & $<1$ & $<1$ & $<1$ & $<1$ \\
\hline alcohol isoamilico & $\begin{array}{c}\text { alcoholico en } \\
\text { bajas } \\
\text { concentraciones } \\
\text { frutado y vinoso }\end{array}$ & $30000 \mathrm{sv}$ & 7 & 10 & 15 & 12 & 8 & 9 & 8 & 9 \\
\hline alcohol isobutílico & $\begin{array}{l}\text { aceite de fusel, } \\
\text { químico }\end{array}$ & $94000 \mathrm{sv}$ & 1 & $<1$ & 1 & 1 & $<1$ & $<1$ & $<1$ & $<1$ \\
\hline $\begin{array}{l}\text { alcohol beta- } \\
\text { feniletilo }\end{array}$ & floral, rosa, miel & $5000 \mathrm{sv}$ & 7 & 8 & 12 & 7 & 8 & 4 & 4 & 4 \\
\hline hexanoato de etilo & $\begin{array}{l}\text { dulce, vinoso, } \\
\text { frutado }\end{array}$ & $5 \mathrm{sv}$ & 26 & 34 & 47 & 35 & 32 & 39 & 48 & 63 \\
\hline butirato de etilo & manzana & $20 \mathrm{sv}$ & $<1$ & 2 & 3 & 4 & 4 & 8 & 8 & 7 \\
\hline acetato de etilo & $\begin{array}{c}\text { en altas } \\
\text { concentraciones, } \\
\text { esmalte de uñas }\end{array}$ & 10000 sv (ref) & 15 & 5 & 2 & 3 & 3 & 5 & 3 & 3 \\
\hline $\begin{array}{l}\text { acetato de } \\
\text { isobutilo }\end{array}$ & $\begin{array}{c}\text { frutado, banana, } \\
\text { manzana, } \\
\text { frambues, pera }\end{array}$ & $73 \mathrm{a}$ & 1 & $<1$ & $<1$ & $<1$ & $<1$ & $<1$ & 1 & $<1$ \\
\hline $\begin{array}{l}\text { acetato de } \\
\text { isoamilo }\end{array}$ & banana & $30 \mathrm{sv}$ & 22 & 19 & 28 & 26 & 18 & 68 & 76 & 39 \\
\hline $\begin{array}{l}\text { acetato de beta- } \\
\text { feniletilo }\end{array}$ & $\begin{array}{l}\text { frutado, anana, } \\
\text { manzana, pera, } \\
\text { banana }\end{array}$ & $250 \mathrm{sv}$ & $<1$ & 4 & $<1$ & $<1$ & $<1$ & 1 & $<1$ & $<1$ \\
\hline$\gamma$-butirolactona & $\begin{array}{l}\text { caramelo, } \\
\text { tostado, } \\
\text { quemado }\end{array}$ & $1000 \mathrm{sv}$ & $<1$ & $<1$ & $<1$ & $<1$ & 1 & $<1$ & $<1$ & $<1$ \\
\hline guaiacol & $\begin{array}{l}\text { hospital, } \\
\text { ahumado, } \\
\text { especiado }\end{array}$ & $9,5 \mathrm{sv}$ & $<1$ & 3 & 20 & 10 & 12 & 23 & 9 & 15 \\
\hline 4-vinil-guaiacol & $\begin{array}{l}\text { clavo de olor, } \\
\text { curry }\end{array}$ & $40 \mathrm{sv}$ & $<1$ & $<1$ & 2 & 3 & 5 & 3 & 2 & 1 \\
\hline 2,6-dimetoxi-fenol & ahumado, nuez & 570 sv & $<1$ & $<1$ & 1 & $<1$ & 1 & 1 & $<1$ & 1 \\
\hline acetaldehído & pungente, eter & 7500 sv (ref) & 1 & 1 & $<1$ & $<1$ & $<1$ & 1 & $<1$ & $<1$ \\
\hline metionol & $\begin{array}{c}\text { repollo } \\
\text { fermentado }\end{array}$ & $500 \mathrm{sv}$ & $<1$ & 2 & 2 & 2 & 2 & $<1$ & $<1$ & 1 \\
\hline
\end{tabular}




\section{REFERENCIAS}

1. P. Ribereau-Gayon, Y. Glories, A. Maujean, D. Dubourdieu, Traité d'oenologia. 2 - Chimie du vin. Stabilisation et traitements. Dunod, Paris (1998)

2. E. Boido, Modificaciones producidas por la fermentación maloláctica en la fracción aromática de los vinos Tannat. Ph D Thesis, Universidad de la República, Montevideo, Uruguay (2002)

3. P. Henschke, 7th Australian Wine Industry Technical Conference, (Williams PJ, Davidson DM, Lee TH. eds), 157-165, Australia (1997)

4. N.P. Jolly, C. Varela, I.S. Pretorius, FEMS Yeast Res. 14 (2), 215-237 (2014)

5. F. Carrau, K. Medina, L. Fariña, E. Boido, E. Dellacassa, Int. J. Food Microbiol. 143, 81-85 (2010)

6. S. Carlin, Metodi di arricchimiento di composti dell' aroma per una possibilite tipicizzazione di vini Traminer, Facoltá de Agraria, Udine, Universitá degli Studi di Udine:98 (1998)

7. E. Boido, A. Lloret, K. Medina, L. Fariña, F. Carrau, G. Versini, E. Dellacassa, J. Agric. Food Chem. 51, 5408-5413 (2003)

8. K. Medina, Biodiversidad de levaduras no-Saccharomyces: Efecto del metabolismo secundario en el color y aroma de vinos de calidad. Ph D Thesis, Universidad de la República, Montevideo, Uruguay (2014)

9. E. Risvik, J.A. McEwan, S. Colwill, R. Rogersa, D.H. Lyonb, Food Qual. Prefer. 5, 263-269 (1994)

10. P. Varela, G. Ares, Food Res. Int. 48, 893-908 (2012)

11. J. Pagès, Food Qual. Prefer. 16, 642-664 (2005)

12. L. Perrin, R. Symoneaux, I. Maître, C. Asselin, F. Jourjon, J. Pagès, Food Qual. Prefer. 19, 1-11 (2008)

13. ISO Sensory analysis: General guidance for the design of test rooms, ISO 8589 (1988)

14. S. Lê, J. Josse, F. Husson, J. Stat. Softw. 25 (1), 1-18 (2008)

15. J.A. Moreno, L. Zea, L. Moyano, M. Medina, Food Control 16, 333 (2005) 